\title{
Ka-band Monolithic GaAs PHEMT Circuits for Transceiver Applications
}

\author{
Chun-Hsien Lien, Kuo-Liang Deng, Chieh-Chao Liu, Hua-Shan Chou, Huei Wang* \\ Department of Electrical Engineering and Graduate Institute of Communication Engineering, \\ National Taiwan University, Taipei, Taiwan 10617, Republic of China \\ E-mail: hueiwang@ew.ee.ntu.edu.tw
}

\begin{abstract}
This paper presents the development of Ka-band circuits for transceiver applications including a low noise amplifier (LNA), a medium power amplifier (PA), a voltagecontrolled oscillator (VCO), and a mixer. These monolithic microwave/millimeter-wave integrated circuits (MMICs) are fabricated with a $0.2-\mu \mathrm{m}$ pseudomorphic (PM) GaAsbased HEMT technology, carried out by commercially available foundry. The LNA demonstrated a measured small signal gain of from 30 to $40 \mathrm{GHz}$ of $17 \mathrm{~dB}$ and flat gain is observed. The PA showed a measured small signal gain $15 \mathrm{~dB}$ at $31 \mathrm{GHz}$. The VCO demonstrated a measured output power $11 \mathrm{dBm}$ at $34.3 \mathrm{GHz}$, and mixer has a measured conversion loss $10.5 \mathrm{~dB}$. Due to fabrication with the commercial foundry process, these MMICs have the potential for mass production.
\end{abstract}

\section{Introduction}

Microwave low noise amplifiers (LNAs), power amplifiers (PAs), voltage-controlled oscillators (VCOs), and mixers are very important components for RF front-end receiver applications. As the wireless communications become more popular, the application frequency is moving toward higher frequency range due to the crowdness of low frequency spectrum. Owing to the advancement of GaAs HEMT MMIC technology [1]-[3], it is feasible to develop MMIC wireless applications. These MMIC chips are fabricated using a commercial $0.2-\mu \mathrm{m}$ pseudomorphic (PM) GaAs-based HEMT technology foundry process [4], therefore they have the potential for mass production. Moreover, these MMIC chips are suitable for front-end low noise receiver applications and can be integrated with other components to form a single-chip RF subsystem.

The LNA has a measured small signal gain of from 30 to $40 \mathrm{GHz}$ is $17 \mathrm{~dB}$ and good gain flatness is observed. The medium PA has measured a small signal gain of $15 \mathrm{~dB}$ at $31 \mathrm{GHz}$. The VCO demonstrated a measured output power of $11 \mathrm{dBm}$ at $34.3 \mathrm{GHz}$. The mixer has a measured conversion loss of $10.5 \mathrm{~dB}$.

\section{Device Characteristics and MMIC Process}

The GaAs-based pseudormorphic HEMT (PHEMT) MMIC process foundry service is provided by Philips Microwave Limeil, France [4]. The device is a $0.2-\mu \mathrm{m}$ gate-length low noise PHEMT with a maximum unit current gain frequency $\left(f_{T}\right)$ of $53 \mathrm{GHz}$. The drain current at peak transconductance under 3-V drain bias is $208 \mathrm{~mA} / \mathrm{mm}$. The passive components include GaAs bulk resistor, MIM capacitor, and via hole through $100-\mu \mathrm{m}$ GaAs substrate. The entire chip is also protected by siliconnitride passivation for reliability concern.

\section{Low Noise Amplifier}

This work was supported in part by the National Science Council, ROC, under Grant NSC 88-2213-E-002- 058 *Correspondence: Email: hueiwang@ew.ee.ntu.edu.tw; Telephone: 886223635251 ext 317; Fax: 88622368 3824 
The two-stage grounded CPW low noise amplifier is designed using 120- $\mu \mathrm{m}$ PHEMT devices [5]. Inductive $\mathrm{T}$ transformers were used as matching networks. MIM capacitors were used for dc blocking and RF bypassing, while air bridges and via holes were fabricated to prevent the excitation of the slot-line modes. Fig. 1(a) shows the chip photo, while the chip size is $2.25 \times 1 \mathrm{~mm}^{2}$. The measured results of the two-stage LNA are shown in Fig. 1(b). Small signal gain from 30 to $40 \mathrm{GHz}$ is $17 \mathrm{~dB}$ and good gain flatness is observed. In the central frequency, both input and output return loss are below $10 \mathrm{~dB}$.

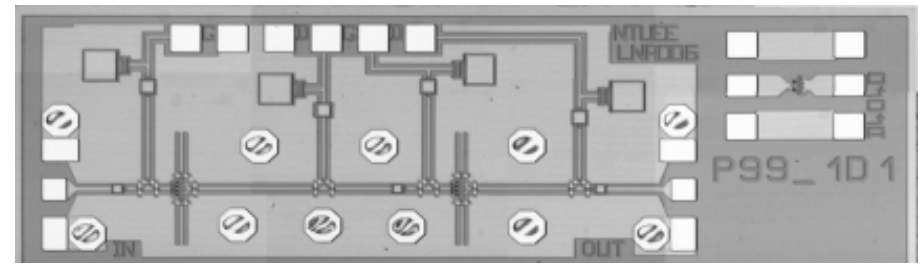

(a)

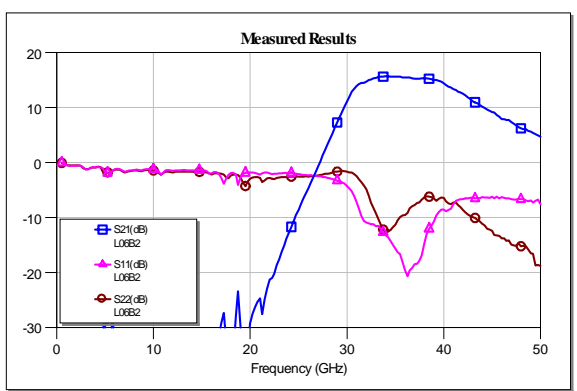

(b)

Fig. 1(a) The chip photo, and (b) the measured results of the two-stage CPW LNA.

\section{Medium Power Amplifier}

This medium power amplifier is a two-stage single-ended microstrip-line design with output device periphery of $360 \mu \mathrm{m}$ [6]. Optimum load at $35 \mathrm{GHz}$ was determined from the device nonlinear model using harmonic balance simulation. Two devices were combined at the second stage to provide enough power and the first stage was designed to drive the second stage. Source inductance is added at driver stage for stability concern. Odd mode clamping resistor was inserted between combined devices to suppress odd mode oscillation. Out-of-band stability was improved by the resistively loaded quarter-wave-length stubs. Fig. 2(a) shows the chip photo of the two-stage PA. Small signal measured results are shown in Fig. 2(b). 15-dB small signal gain is achieved at $31 \mathrm{GHz}$.

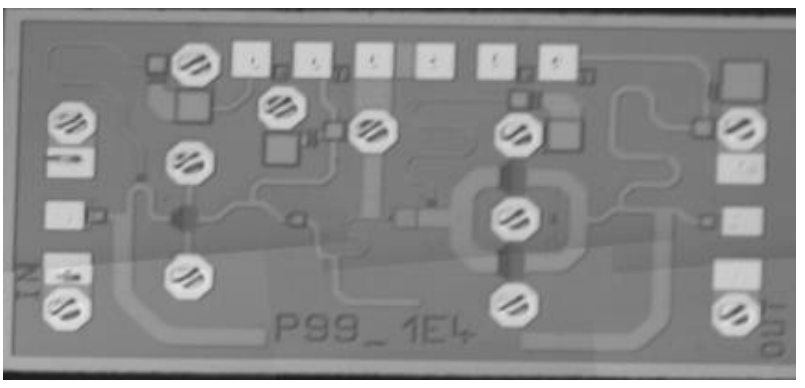

(a)

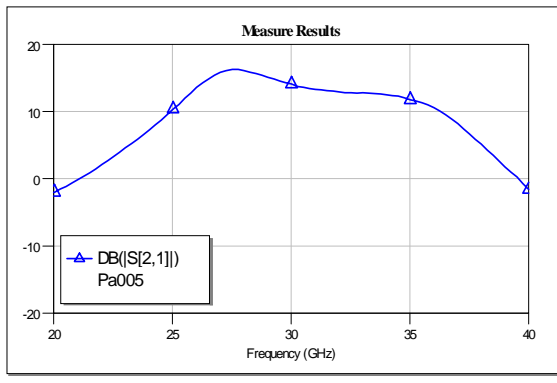

(b)

Fig. 2(a) The chip photo. (b) The measured results of the two-stage microstrip-line PA.

\section{Voltage-Controlled Oscillator}

A four-finger with $120-\mu \mathrm{m}$ periphery PHEMT was used for the output power consideration. The $\mathrm{VCO}$ is a common gate design, and the oscillating frequency is tuned by the gate voltage. A pair of coupled lines were used to couple the output power to the output port and served as a dc blocking. Fig. 3(a) shows the chip photo of the monolithic 35-GHz VCO, with a chip size of $2.25 \times 1 \mathrm{~mm}^{2}$. Fig. 3 (b) shows the spectrum plot of the output signal. An 11-dBm output power is measured at $34.3 \mathrm{GHz}$. The output frequency can be tuned by using the gate bias. The tuning range is $330 \mathrm{MHz}$. 


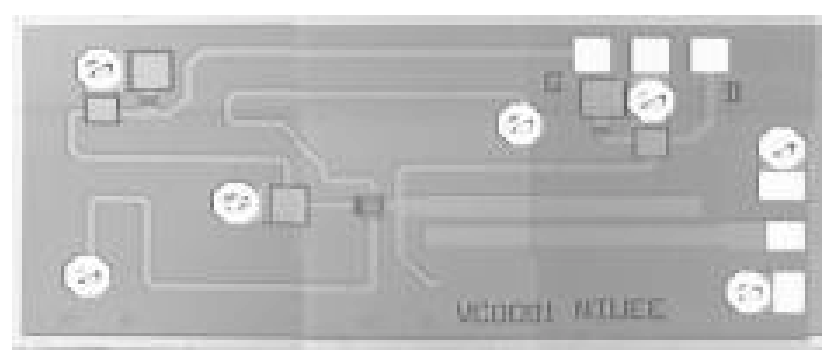

(a)

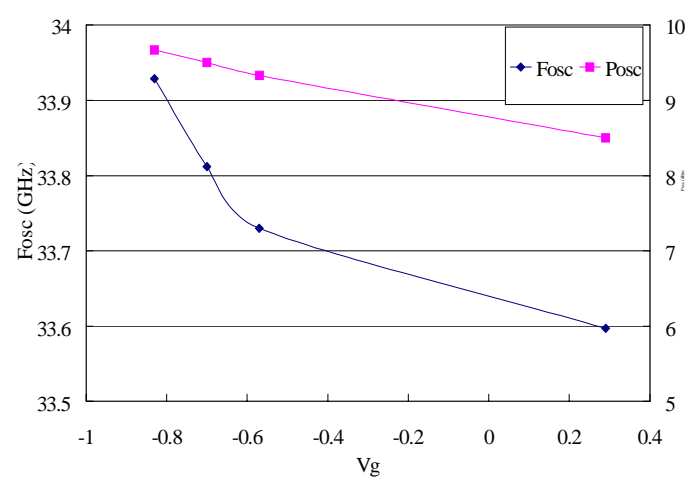

(b)

Fig. 3(a) the chip photo, and (b) output power and tuning range as function of bias voltage of the VCO.

\section{Mixer}

The singly balanced diode mixer was designed using microstrip-lines. The Lange coupler was selected as $90^{\circ} 3-\mathrm{dB}$ hybrid for bandwidth consideration. A pair of four-finger diode with $80-\mu \mathrm{m}$ were selected. A low pass filter was designed to achieve both Lo-to-IF and RF-to-IF isolations. Fig. 4(a) shows the chip photo of this mixer, while the chip size is $1.5 \times 1 \mathrm{~mm}^{2}$. About $10.5 \mathrm{~dB}$ conversion loss is achieved as shown in Fig. 4(b). Isolation and return loss measurements are shown in Fig. 4 (c) and Fig. 4(d) receptively. The return loss of LO and RF are both better than $20 \mathrm{~dB}$ and isolation are better than $10 \mathrm{~dB}$ and $25 \mathrm{~dB}$.

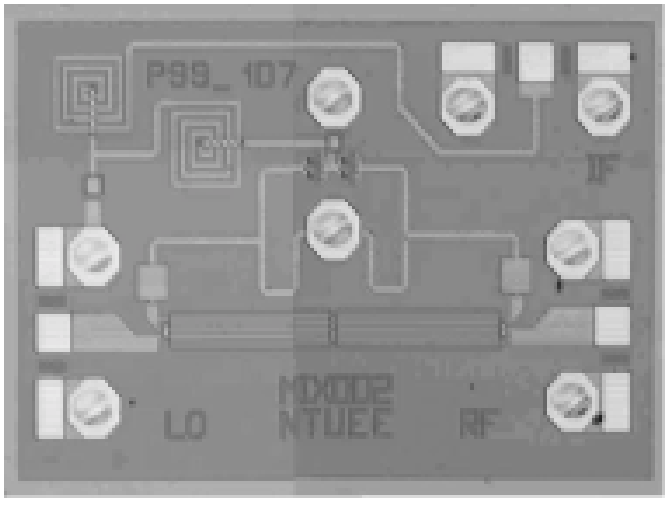

(a)

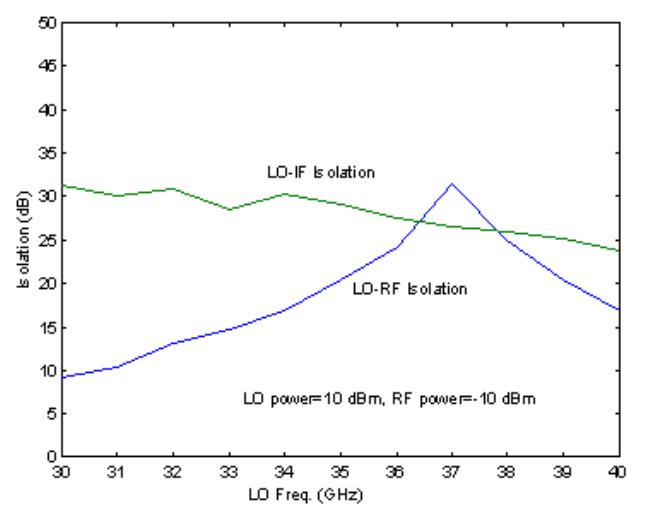

(c)

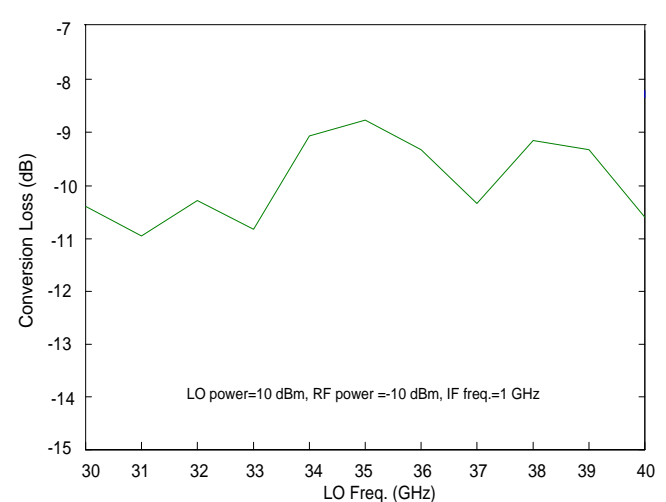

(b)

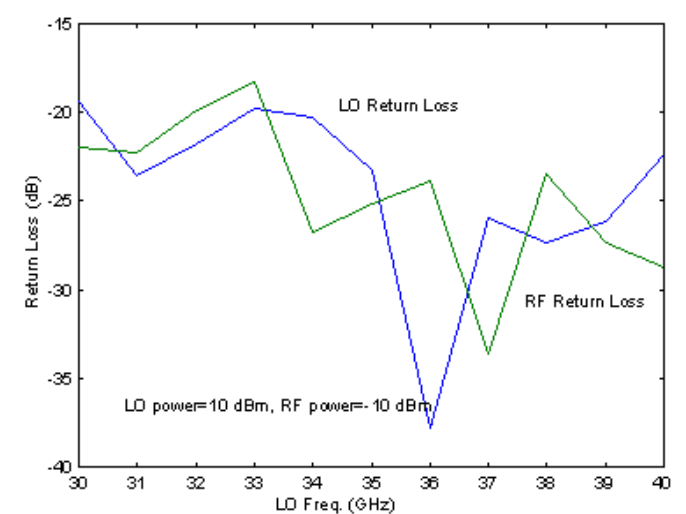

(d)

Fig. 4 The measured results of the singly balanced diode mixer. (a) chip photo (b) conversion loss (c) isolation (d) return loss 


\section{Summary}

Several Ka-band monolithic chips including a CPW LNA, a medium PA, a VCO, and a singly balanced-diode mixer for RF front-end receiver applications were developed with reasonable performance. The MMIC chips are fabricated via commercial GaAs PHEMT foundry process and these chips can be integrated with other RF transceiver components into a multi-functional chip for compact transceiver module applications.

\section{Acknowledgements}

The authors would like to acknowledge Dr. D. C. Niu of Chung-Shan Science and Technology Institute for his valuable suggestions and help in the testing, Dr. K. W. Huang of Nano Device Laboratory for their help on measuring. Thanks also go to Chip Implementation Center of Taiwan for their foundry service coordination effort.

\section{References}

[1] B. Nelson, et al., "Octave band InGaAs HEMT MMIC LNA's to $40 \mathrm{GHz}$," 1990 IEEE GaAs IC Symposium Digest, pp. 165-168, Nov., 1990.

[2] D. Ingram, et al., "A highly integrated multi-functional chip set for low cost Ka-band transceiver," 1998 IEEE MTT-S International Microwave Symposium Digest, vol. 1, pp. 301-304, June, 1998.

[3] Huei Wang, T. H. Chen, D. C. Niu, Y. J. Chan, P. W. Kwo, H. S. Chou, and K. L. Deng, " Ka-band Monolithic GaAs PHEMT Low Noise Amplifiers for Commercial Wireless Applications," Conference Proceedings of Wireless Technologies and Systems: Millimeter Wave and Optical, part of SPIE's International Symposium on Voice, Video, and Data Communications, Boston, MA, Sept., 1999.

[4] Philips Microwave D02AH V2.0 Design Manual, Phlips Microwave Lemeil, Jan., 1997.

[5] H. S. Chou, "Design of 2.4 and 35-GHz low noise amplifiers," Master Thesis, National Taiwan University, June 1999.

[6] C. C. Liu, "Design of 2.4 and 35-GHz power amplifiers," Master Thesis, National Taiwan University, June 1999. 